\title{
Description of van der Waals Interactions Using Transformation Optics
}

\author{
Rongkuo Zhao, ${ }^{*}$ Yu Luo, A. I. Fernández-Domínguez, and J. B. Pendry \\ The Blackett Laboratory, Department of Physics, Imperial College London, London SW7 2AZ, United Kingdom
}

(Received 12 May 2013; published 17 July 2013)

\begin{abstract}
Exact calculation of the van der Waals interaction between closely spaced plasmonic nanoparticles is challenging due to the strong concentration of the electromagnetic fields that takes place at the nanometric gap between them. The technique of transformation optics, capable of mapping a small volume into any desired length scale, enables us to shed physical insight into the intricate behavior of electromagnetic fields in extremely small gaps. Using this theoretical tool, we obtain universal analytical expressions for the van der Waals interactions between spherical nanoparticles made of realistic metals at arbitrary separation.
\end{abstract}

DOI: 10.1103/PhysRevLett.111.033602

PACS numbers: 42.50.Lc, 03.70.+k, 12.20. $-\mathrm{m}$

van der Waals (vdW) forces between nanostructures, which arise from the electromagnetic interaction between instantaneous quantum fluctuation-induced charges, play a key role in surface adhesion [1,2] and friction [3], colloidal stability and crystallization [4], nanoparticle self-assembly $[5,6]$, and supramolecular (viruses, proteins, polymers) interactions. A quantitative description of these quantum forces is of great importance to a broad range of areas such as surface and colloidal science, nanocomposite engineering, nanotechnology, and macromolecular chemistry. vdW forces can be calculated by asymptotic equations [7] in the limiting cases of small molecules and large macroscopic bodies. However, the calculation of $\mathrm{vdW}$ forces between nanoparticles whose size and separation are comparable is complicated. Traditional procedures via summing over infinitesimal elements, such as Hamaker's method [8] and the proximity force approximation (PFA) [9], have been widely used for estimating the $\mathrm{vdW}$ interactions in different geometries [7]. However, vdW forces are inherently nonadditive interactions. This fact makes the aforementioned methods not valid for the collective interaction between nanoparticles. Using transformation optics we have recently developed a powerful formalism to calculate the spectra of singular and near singular structures [10]. Here we present details of its application to calculate vdW forces for the difficult problem of two nearly touching spheres: exactly in the absence of retardation, and in the presence of retardation to an accuracy of $4 \%$ over the entire range of particle separations.

If two metallic nanoparticles are separated by a small distance, the excitation of surface plasmons in the system leads to a strong concentration of the electromagnetic fields at the nanometric gap [11-19]. Hence, this portion of the fields dominates the mechanical interactions between the nanoparticles. The exact calculation of $\mathrm{vdW}$ forces requires a knowledge of the spectrum arising from the mutual electromagnetic scattering between the curved nanoparticle surfaces at the gap. Multipole expansion [20-22] and full numerical simulations [23] have been used to describe the scattering properties of such systems.
In order to capture the strong field confinement in tiny volumes, a large number of multipole moments [22,24] or extremely fine meshes [25] are required. For instance, in a sphere-plane configuration with a gap-to-radius ratio of $10^{-3}$, several thousand spherical harmonics are needed [24]. vdW forces between ideal metallic spheres can be given asymptotically using bispherical coordinates in the lossless limit [26]. However, for more realistic treatments, the calculation remains challenging. The powerful technique of transformation optics [27-30], capable of mapping a near touching geometry into any desired separation, enables us to shed physical insight into the intricate behavior of electromagnetic fields at extremely small metallic gaps [31]. Through the extension of the transformation optics framework to three-dimensional geometries, we have recently developed accurate and extremely efficient approaches for calculating the absorption and scattering spectra, field enhancement performance, modal frequencies, and spatial distribution for nanosphere dimers and spherical crescents $[10,32,33]$.

In this Letter, we study in detail the vdW forces between two spheres and between a sphere and a plane, with arbitrary separation. The permittivity is modeled using realistic silver parameters. The physical mechanisms behind the $\mathrm{vdW}$ forces between plasmonic nanoparticles are revealed through a comprehensive analysis of the eigenmodes supported by these systems. Finally, we obtain accurate approximate analytic expressions for the vdW forces in both the sphere-sphere and sphere-plane geometries. We work within the quasistatic limit, so the structure size is assumed to be much smaller than the wavelength of the virtual photons responsible for the force. Note that the latter is determined by the spectral characteristics of the material (indicated by the plasmon frequency in the case of metals). For large separations, retardation effects can be taken into account through correction of the dipole contributions.

Taking a conformal inversion transformation [10] of the form $\mathbf{r}-\mathbf{R}_{0}=R_{T}^{2}\left(\mathbf{r}^{\prime}-\mathbf{R}_{0}^{\prime}\right) /\left|\mathbf{r}^{\prime}-\mathbf{R}_{0}^{\prime}\right|^{2}$, two spheres of radii $R_{1}^{\prime}$ and $R_{2}^{\prime}$ separated by a gap distance $\delta^{\prime}$ can be mapped into an annulus comprising two concentric spheres 
of radii $R_{1}$ (inner solid one) and $R_{2}$ (outer hollow one). See Fig. $1 . R_{T}^{2}$ is an arbitrary length scale parameter and $R_{0}^{\prime}$ $\left(R_{0}\right)$ gives the position of the inversion point in the two spheres (annulus) frame. The annulus can be transformed back to the two spheres by performing a second inversion. The annulus radii can be expressed in terms of the spheres' geometric parameters through $2 R_{1}=R_{T}^{2} /\left(\delta^{\prime}+d^{\prime}\right)-d_{1}$ and $2 R_{2}=R_{T}^{2} / d^{\prime}-d_{2}$, where, $d_{1}=R_{T}^{2} /\left(2 R_{1}^{\prime}+\delta^{\prime}+d^{\prime}\right)$, $d_{2}=R_{T}^{2} /\left(2 R_{2}^{\prime}-d^{\prime}\right)$, and $d^{\prime}=R_{2}^{\prime}-R_{0}^{\prime}$.

The tiny gap between the two spheres in Fig. 1(a) is mapped into a larger region located further from the inversion point in Fig. 1(b). Note that the annulus geometry has higher symmetry. Inherently to this geometric simplification, the permittivity becomes inhomogeneous, having $\epsilon(\mathbf{r})=R_{T}^{2}\left|\mathbf{r}-\mathbf{R}_{0}\right|^{-2} \epsilon^{\prime}\left(\mathbf{r}^{\prime}\right)$. Note that in the following we assume that the two spheres in Fig. 1(a) have the same permittivity, $\epsilon_{1}^{\prime}=\epsilon_{2}^{\prime}=\epsilon^{\prime}$, and are embedded in a medium of dielectric constant $\epsilon_{D}^{\prime}$. Despite this complication, the theoretical treatment of the two spheres is greatly facilitated by the transformation into the annulus frame. The general solutions of the potential $\varphi(\mathbf{r})$ in Fig. 1(b) are of the form

$\varphi_{l m}=\left|\mathbf{r}-\mathbf{R}_{0}\right|\left[a_{l m}^{+}\left(r / R_{0}\right)^{l}+a_{l m}^{-}\left(R_{0} / r\right)^{l+1}\right] Y_{l m}(\theta, \phi)$,

where $l \geq 0$ and $|m| \leq l$. $Y_{l m}$ are spherical harmonics. $a_{l m}^{ \pm}$ are unknown expansion coefficients determined by the electromagnetic boundary conditions. Through this field matching, the solution with total angular momentum $l$ couples only to $l \pm 1$ components thanks to the $\cos \theta$ term inherent to the $\left|\mathbf{r}-\mathbf{R}_{0}\right|$ factor in Eq. (1). This results in a tridiagonal system of equations. The azimuthal angular momentum $m$ is conserved under the transformation. In order to ensure that the total charge accumulated in each

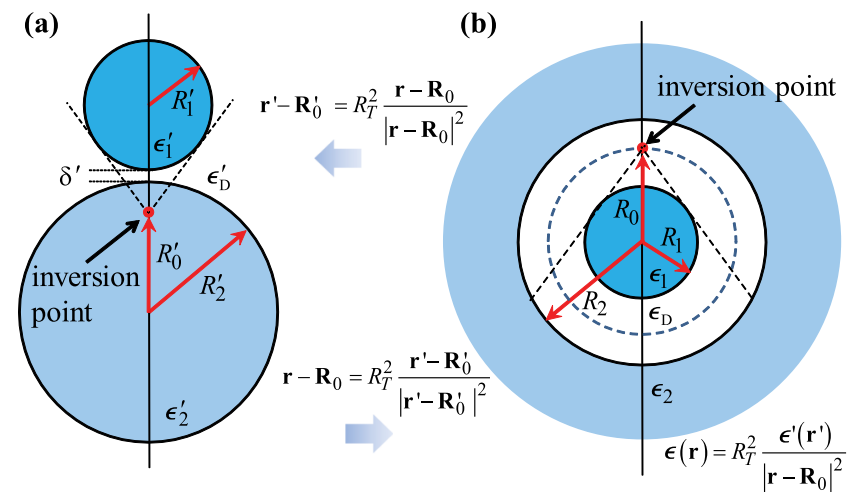

FIG. 1 (color online). Applying an inversion transformation about the point $\mathbf{R}_{0}^{\prime}$, two separated spheres (a) are mapped into an annulus (b), and vice versa. The annulus geometry comprises an inner solid sphere and an outer hollow one. The geometric inversion is accompanied by a transformation of the system permittivities. The small gap inside the cone indicated by the two dashed lines in (a) is enlarged in the annulus and is located further from the inversion point in (b). The opening angle of the cone in (a) is unchanged under the conformal transformation. sphere must be equal to zero, we impose an additional condition on the $m=0$ coefficients $a_{l 0}^{ \pm}$,

$$
\sum_{l=0}^{+\infty} \sqrt{2 l+1} a_{l 0}^{ \pm}=0 .
$$

The multipole expansion [20-22] for vdW force calculations needs a translation matrix that transfers a solution expressed in a basis appropriate to one object to a basis appropriate to the other. The matrix is usually neither unitary nor diagonal, as it depends on the displacement and orientation of the two coordinate bases. However, in the annulus system in Fig. 1(b), the fields scattered by each sphere are expanded about the same origin: see Eq. (1). Therefore, a translation matrix is not needed in the annulus geometry leading to a simpler calculation. A related approach using bispherical coordinates [34] also yields a unit translation matrix.

In such a situation, the total vdW energy can be directly expressed in terms of the reflection amplitudes (similar to the two parallel surfaces geometry [35]) of the inner and outer spheres evaluated at the spherical surface indicated by the dashed circle in Fig. 1(b). Thus, we can write

$$
U=\frac{\hbar}{4 \pi i} \sum_{m=0}^{+\infty} \int_{-\infty}^{+\infty} \ln \operatorname{det}\left(\mathbf{1}-\mathbf{R}_{m}^{-} \mathbf{R}_{m}^{+}\right) d \omega,
$$

where $\mathbf{R}_{m}^{ \pm}=\left(\mathbf{T}_{m}^{ \pm \mp}\right)^{-1} \mathbf{S}_{m}^{ \pm \pm}$are the reflection matrices for the inner (upper superscript) and outer (lower superscript) spheres. $\mathbf{T}_{m}^{ \pm \overline{ }}$ and $\mathbf{S}_{m}^{ \pm \pm}$are tridiagonal matrices given by

$$
\begin{aligned}
S_{l, l-1}^{ \pm \pm} & =(2 l-1) p_{l}^{m} \chi_{ \pm}^{-2}, \\
S_{l, l}^{ \pm \pm} & =-\left(l+1+l \chi_{ \pm}^{-2}\right), \\
S_{l, l+1}^{ \pm \pm} & =(2 l+3) p_{l+1}^{m},
\end{aligned}
$$

$$
\begin{aligned}
T_{l, l-1}^{ \pm \mp} & =-e^{-\alpha} S_{l, l-1}^{ \pm \pm} \chi_{ \pm}^{-2 l+1}, \\
T_{l, l}^{ \pm \mp} & =\left[\frac{\chi_{ \pm}^{-2}-1}{\epsilon^{\prime}-1}-e^{-\alpha} S_{l, l}^{ \pm \pm}\right] \chi_{ \pm}^{-2 l-1}, \\
T_{l, l+1}^{ \pm \mp} & =-e^{-\alpha} S_{l, l+1}^{ \pm \pm} \chi_{ \pm}^{-2 l-3},
\end{aligned}
$$

where $e^{\alpha}=\left(\epsilon^{\prime}-\epsilon_{D}^{\prime}\right) /\left(\epsilon^{\prime}+\epsilon_{D}^{\prime}\right), \chi_{+}=R_{1} / R_{0}, \chi_{-}=R_{0} / R_{2}$, and $p_{l}^{m}=\sqrt{\left(l^{2}-m^{2}\right) /\left(4 l^{2}-1\right)}$. In actual computations, the matrices are truncated at $l=l_{\max }$ so that they have dimensions $\left(l_{\max }-m+1\right) \times\left(l_{\max }-m+1\right)$ for each $m$. For $m=0$, the zero charge condition given by Eq. (2) should be applied to both $\mathbf{T}_{m}^{ \pm \mp}$ and $\mathbf{S}_{m}^{ \pm \pm}$. Mathematically, this procedure consists of the introduction of an extra row,

$$
[\sqrt{1}, \ldots, \sqrt{2 l-1}, \sqrt{2 l+1}, \sqrt{2 l+3}, \ldots],
$$

which is the eigenvector of the net charge mode [10]. This cancels the elements in the first columns of $\mathbf{T}_{m}^{ \pm \mp}$ and $\mathbf{S}_{m}^{ \pm \pm}$, ensuring charge conservation. Importantly, the sparse matrices obtained this way are very easy to manipulate. 
Exact results.-To illustrate the power of our approach, we apply it to the study of the vdW energies between two identical spheres and between a sphere and a plane in vacuum. The dielectric properties of all the metal structures are modeled using the Palik data for silver [36]. In the following, the prime for the original frame in Fig. 1(a) is omitted for convenience.

The poles of the integrand in Eq. (3) within the complex plane yield complex frequencies for the eigenmodes supported by the system. These are given by the roots of

$$
\operatorname{det}\left(\mathbf{1}-\mathbf{R}_{m}^{-} \mathbf{R}_{m}^{+}\right)=0 .
$$

The eigenmode frequencies versus gap distance for a sphere-sphere (upper panels) and a sphere-plane (lower panels) geometries are shown in Fig. 2. For clarity, losses are omitted so that the poles lie along the real axis in the complex frequency plane. In both structures, the modes can be classified into two groups. One group [light gray (blue) dots] shifts to lower frequencies as the gap decreases and contributes to the attractive forces between the two objects. The other [dark gray (red) dots] goes to higher frequencies, yielding a repulsive interaction. The total $\mathrm{vdW}$ force is the result of the competition between these two groups.

In principle, the vdW energy can be calculated by the summation of the zero point energy $\hbar \omega_{i} / 2$ over all the complex modes [26,37]. But, in practice, the introduction of absorption losses complicates greatly the identification of the complex roots of Eq. (7). This fact makes the integration method given by Eq. (3) the most convenient. Note that the integration can be easily evaluated along the imaginary axis by rotating the contour. In order to ensure

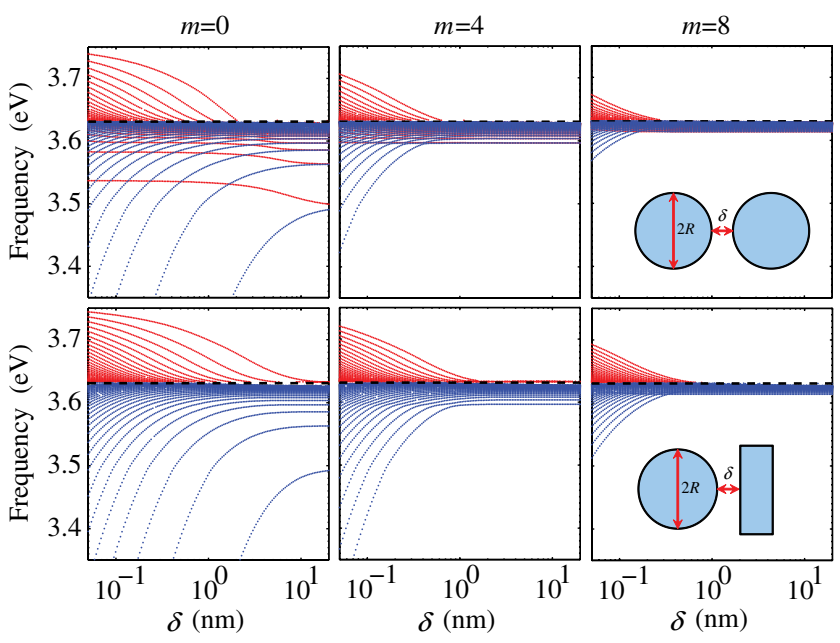

FIG. 2 (color online). Eigenmodes of two identical spheres (upper panels) and of a sphere and a plane (lower panels) for different azimuthal angular momenta, $m=0,4,8$ (first, second, third columns, respectively). The loss of silver is omitted for clarity. The surface plasmon frequency at $3.63 \mathrm{eV}$ is indicated by the black dashed line in all panels. In all panels, $R=5 \mathrm{~nm}$. Note that these results are scale invariant in the quasistatic approximation. the validity of the contour rotation, we fit Palik's data for silver [36] using one plasmonic Drude model with damping plus six modified Lorentz terms. This fitting function automatically satisfies the Kramers-Kronig relation and is analytic in the upper half-plane (see Supplemental Material [38] for the fitting details).

Figure 3(a) shows the fully converged results of the vdW energy between two identical spheres and between a sphere and a plane as a function of distance. At $\delta / R=0.1(0.01)$ the vdW energy of the sphere-sphere geometry is 1.2 (17.8) $\mathrm{eV}$ and of the sphere-plane geometry 2.9 (36.4) eV. The room temperature kinetic energy of a Brownian particle, $k_{B} T / 2=0.013 \mathrm{eV}$, is indicated by a black dashed line. The vdW energies exceed this value when $\delta / R<0.42$ for the sphere-sphere geometry and $\delta / R<0.86$ for the sphere-plane case, which explains the phenomena of adhesion and self-assembly in nanoparticles.

In the small gap limit, the vdW energy of the sphereplane geometry is roughly twice the energy of the spheresphere configuration. In this region, both vdW energies are proportional to $(\delta / R)^{-1}$. In the large gap limit, the sphere-plane energy decays much faster than the spheresphere one, with characteristic decays $(\delta / R)^{-3}$ and $(\delta / R)^{-6}$, respectively.

The lack of a "singular" gap in the annulus system makes the fast convergence of our approach possible, without the requirement of a large number of partial waves. For a gap-to-radius ratio $\delta / R=10^{-2}\left(10^{-3}\right)$ in the sphereplane geometry, only $35(\sim 100)$ partial waves are need to achieve a $99.99 \%$ accuracy [Fig. 3(b)]. We define the accuracy in terms of the ratio $U\left(l_{\max }\right) / U\left(l_{\max }+1\right)$, where $U\left(l_{\max }\right)$ gives the vdW energy obtained for a maximum partial wave order equal to $l_{\max }$. This result clearly shows that our transformation optics approach converges much

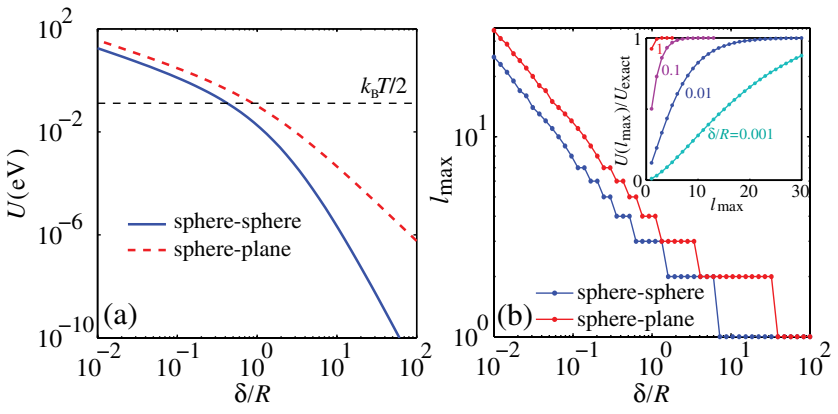

FIG. 3 (color online). (a) The fully converged vdW energy between two silver spherical nanoparticles and between a sphere and a plane as a function of the gap distance. The characteristic kinetic energy of Brownian particle $k_{B} T / 2$ at room temperature is indicated by the black dashed line. (b) The maximum partial wave order $l_{\max }$ needed to realize $99.99 \%$ accuracy defined by the ratio $U\left(l_{\max }\right) / U\left(l_{\max }+1\right)$ as a function of the gap size. The inset in (b) renders the vdW energy of the sphere-plane geometry as a function of $l_{\max }$ normalized to the exact one and for different gap-to-radius ratio. Note that the contributions of different $m$ to the total energy have been summed over. 
faster than the multipole expansion method [24] where several thousands of terms are needed for a $10^{-3}$ gap-toradius ratio. The $\mathrm{vdW}$ energy for the sphere-sphere geometry converges even faster than the sphere-plane case.

Although $l$ cannot be used to label each eigenmode, as partial waves for a given $l$ couple with $l \pm 1$ components, we can estimate each mode contribution through the slope of the curve of the vdW energy versus $l_{\max }$ [inset of Fig. 3(b)]. At large separations, $\delta / R \geq 0.01$, the main contribution comes from the lowest angular momenta. At small separations, $\delta / R=0.001$ for instance, it comes from the moderate angular momenta. This reflects the fact that the total number of high order modes is enormous, but the frequency shifts they experience with gap size are rather small. On the other hand, the number of the low order modes is small, but they exhibit significant frequency shifts as a function of the gap size in Fig. 2.

Approximate results and retardation corrections.-As the gap decreases, $\chi_{ \pm}$tends to 1 , so the term $\left(\chi_{ \pm}^{-2}-1\right) /$ $\left(\epsilon^{\prime}-1\right)$ in $T_{l, l}^{ \pm \mp}$ approaches 0 . If these small terms are neglected, the difference between $\mathbf{T}_{m}^{ \pm \mp}$ and $\mathbf{S}_{m}^{ \pm \pm}$is only the prefactors of $e^{-\alpha} \chi_{ \pm}^{-2 l-1}$. Therefore, the reflection matrices $\mathbf{R}_{m}^{ \pm}$become diagonal, except for the $m=0$ case where the charge neutrality condition Eq. (2) has to be imposed on $\mathbf{T}_{m=0}^{ \pm \mp}$ and $\mathbf{S}_{m=0}^{ \pm \pm} . \mathbf{R}_{m=0}^{ \pm}$are still solvable analytically and can be expressed as a diagonal matrix plus a matrix with identical rows. The determinant of such a matrix is given by its trace. For the sphere-sphere geometry, this enables us to write

$$
\begin{aligned}
& \sum_{m=0}^{+\infty} \ln \operatorname{det}\left(\mathbf{1}-\mathbf{R}_{m}^{-} \mathbf{R}_{m}^{+}\right) \\
& \approx \sum_{l=1}^{+\infty}(2 l+1) \ln \left(1-e^{2 \alpha} \chi^{4 l+2}\right) \\
& \quad+\ln \left[1-e^{\alpha} \sum_{l=1}^{+\infty} \frac{\left(1-\chi^{2}\right)\left(1-\chi^{2 l}\right) \chi^{2 l+1}}{1-e^{\alpha} \chi^{2 l+1}}\right] \\
& \quad+\ln \left[1+e^{\alpha} \sum_{l=1}^{+\infty} \frac{\left(1-\chi^{2}\right)\left(1-\chi^{2 l}\right) \chi^{2 l+1}}{1+e^{\alpha} \chi^{2 l+1}}\right],
\end{aligned}
$$

where $\chi=\chi_{+}=\chi_{-}<1$, and

$$
\begin{aligned}
& \sum_{m=0}^{+\infty} \ln \operatorname{det}\left(\mathbf{1}-\mathbf{R}_{m}^{-} \mathbf{R}_{m}^{+}\right) \\
& \approx \sum_{l=1}^{+\infty}(2 l+1) \ln \left(1-e^{2 \alpha} \chi^{2 l+1}\right) \\
& \quad+\ln \left[1-e^{\alpha} \sum_{l=1}^{+\infty} \frac{\left(1-\chi^{2}\right)\left(1-\chi^{2 l}\right) \chi^{2 l+1}}{1-e^{\alpha} \chi^{2 l+1}}\right],
\end{aligned}
$$

for the sphere-plane configuration, with $\chi=\chi_{+}<1$ $\left(\chi_{-}=1\right.$ in this case). In the limit of a perfect conductor $(\epsilon=-\infty)$, Eqs. (8) and (9) become exact and the latter is exactly the same as the result in Ref. [34].
In the small gap limit, our approximations, Eqs. (8) and (9), are more accurate than the PFA; see Fig. 4(a) which plots the relative error for different approximations measured by the magnitude $\left|U / U_{\text {exact }}-1\right|$. For larger gaps, where the PFA results break down, our approximations are still valid. The deviations of our approximate expressions from the exact predictions are less than $17 \%$ for the sphereplane geometry and $35 \%$ for the sphere-sphere case.

The accuracy of our approximate approach at large gaps can be significantly improved through the replacement of the dipole contribution terms, $6 e^{2 \alpha} /(2+\delta / R)^{6}$ in Eq. (8) and $e^{2 \alpha} / 2(1+\delta / R)^{3}$ in Eq. (9) by the well-known correct ones, $6 \Delta^{2} /(2+\delta / R)^{6}$ and $e^{\alpha} \Delta / 2(1+\delta / R)^{3}$, respectively, where $\Delta=(\epsilon-1) /(\epsilon+2)$ [39,40]. The dipole term corrected results are shown in Fig. 4(a). Note that the dipole term corrected predictions are more accurate than the dipole approximations at large separations. Here, by the dipole approximation we mean the small particle limit in which the vdW energy is fully described by dipole interactions. At intermediate separations, the discrepancies between our dipole term corrected results and the exact ones reach their maxima: $4 \%$ at $\delta / R=0.2$ for the sphereplane and $14 \%$ at $\delta / R=0.4$ for the sphere-sphere configuration. Obviously, the accuracy could be further improved through correcting higher order modes as well. We note that our dipole corrected approximation is accurate at separations where the vdW energy is comparable to $k_{B} T$ and therefore has significant advantages in practical situations.

Following a similar procedure, nonretarded dipole contributions can be replaced by retarded ones [40,41]. Thus, retardation effects at large separations can be taken into account [see Fig. 4(b)]. Note that the dipole contribution dominates the interaction at large gap sizes. Even though these "hybrid" retarded vdW energies are not exact, we can estimate from the weight of the dipole
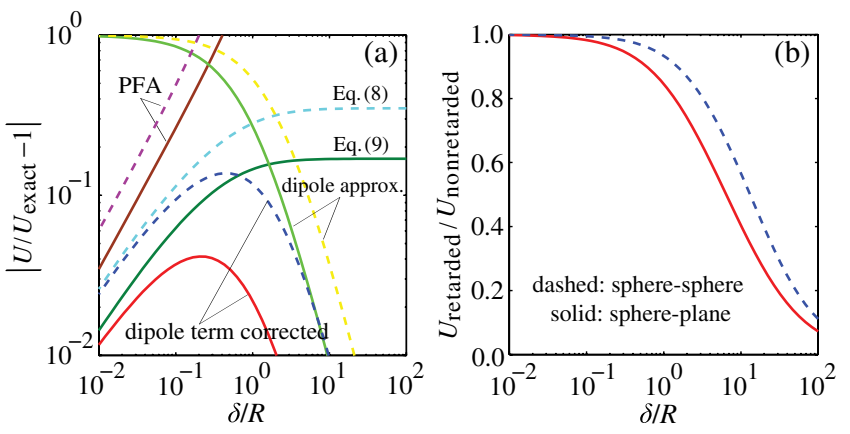

FIG. 4 (color online). (a) Deviations from the exact prediction of different approximations: expressions Eqs. (8) and (9), the PFA [9], the dipole term corrected results, and the dipole approximations. (b) The retarded vdW energy calculated by replacing the nonretarded dipole contribution with the retarded one, normalized to the exact nonretarded vdW energy. Dashed and solid curves in each panel correspond to the sphere-sphere and the sphere-plane geometry, respectively. 
contributions in Fig. 4(a) that they possess at least $90 \%$ accuracy when the gap-to-radius ratio is larger than 2.3 (5.0) for the sphere-plane (sphere-sphere) geometry.

In this Letter we have used transformation optics to study the van der Waals interactions between two plasmonic nanospheres as well as a sphere and a plane. Specifically, we have exploited an inversion transformation that maps these gapped geometries into an annulus one. This unveils the hidden geometric symmetry of the original structure and not only makes the fast convergence of exact calculations possible, but also enables us to obtain accurate analytical approximations.

We thank the following for support: the Gordon and Betty Moore Foundation (A.I.F.-D. and J.B.P.), the AFOSR (J.B.P.), the Royal Commission for the Exhibition of 1851 (R.Z.), and the Leverhulme Trust (Y. L. and J. B.P.).

*r.zhao@imperial.ac.uk

[1] F. W. Delrio, M. P. de Boer, J. A. Knapp, E. D. Reedy, P. J. Clews, and M. L. Dunn, Nat. Mater. 4, 629 (2005).

[2] K. Autumn, K. Autumn, Y. A. Liang, S. T. Hsieh, W. Zesch, W. P. Chan, T. W. Kenny, and R. Fearing, Nature (London) 405, 681 (2000).

[3] Y. Mo, K. T. Turner, and I. Szlufarska, Nature (London) 457, 1116 (2009).

[4] P. C. Hiemenz and R. Rajagopalan, Principles of Colloid and Surface Chemistry (Marcel Dekker, New York, 1997), 3rd ed.

[5] Y. Min, M. Akbulut, K. Kristiansen, Y. Golan, and J. Israelachvili, Nat. Mater. 7, 527 (2008).

[6] Y. Lalatonne, J. Richardi, and M. P. Pileni, Nat. Mater. 3, 121 (2004)

[7] J. N. Israelachvili, Intermolecular and Surface Forces (Academic, San Diego, 1998).

[8] H. C. Hamaker, Physica (Amsterdam) 4, 1058 (1937).

[9] B. V. Deriagin, I. I. Abrikosova, and E. M. Lifshitz, Q. Rev. Chem. Soc. 10, 295 (1956).

[10] J. B. Pendry, A. I. Fernández-Domínguez, Y. Luo, and R. Zhao, Nat. Phys., doi:10.1038/nphys2667 (2013).

[11] R. C. McPhedran and D. R. McKenzie, Appl. Phys. 23, 223 (1980).

[12] R. C. McPhedran and W. T. Perrins, Appl. Phys. 24, 311 (1981).

[13] R. C. McPhedran and G. W. Milton, Proc. R. Soc. A 411, 313 (1987).

[14] F. J. García-Vidal and J. B. Pendry, Phys. Rev. Lett. 77, 1163 (1996).

[15] M. I. Stockman, Phys. Rev. Lett. 93, 137404 (2004).
[16] C. E. Talley, J. B. Jackson, C. Oubre, N. K. Grady, C. W. Hollars, S. M. Lane, T. R. Huser, P. Nordlander, and N. J. Halas, Nano Lett. 5, 1569 (2005).

[17] L. A. Sweatlock, S. A. Maier, H. A. Atwater, J. J. Penninkhof, and A. Polman, Phys. Rev. B 71, 235408 (2005).

[18] I. Romero, J. Aizpurua, G. W. Bryant, and F. Javier García De Abajo, Opt. Express 14, 9988 (2006).

[19] R. T. Hill, J. J. Mock, Y. Urzhumov, D. S. Sebba, S. J. Oldenburg, S.-Y. Chen, A. A. Lazarides, A. Chilkoti, and D. R. Smith, Nano Lett. 10, 4150 (2010).

[20] D. Langbein, J. Phys. Chem. Solids 32, 1657 (1971).

[21] R. Balian and B. Duplantier, Ann. Phys. (N.Y.) 104, 300 (1977); 112, 165 (1978).

[22] T. Emig, N. Graham, R. L. Jaffe, and M. Kardar, Phys. Rev. Lett. 99, 170403 (2007).

[23] A. W. Rodriguez, F. Capasso, and S. G. Johnson, Nat. Photonics 5, 211 (2011), and references therein.

[24] A. Canaguier-Durand, G.-L. Ingold, M.-T. Jaekel, A. Lambrecht, P. A. M. Neto, and S. Reynaud, Phys. Rev. A 85, 052501 (2012).

[25] A. P. McCauley, M. T. Homer Reid, M. Krüger, and S. G. Johnson, Phys. Rev. B 85, 165104 (2012).

[26] V. V. Klimov and A. Lambrecht, Plasmonics 4, 31 (2009).

[27] A. J. Ward and J. B. Pendry, J. Mod. Opt. 43, 773 (1996).

[28] U. Leonhardt, Science 312, 1777 (2006).

[29] J. B. Pendry, D. Schurig, and D. R. Smith, Science 312, 1780 (2006).

[30] J. B. Pendry, Opt. Express 11, 755 (2003).

[31] J. B. Pendry, A. Aubry, D. R. Smith, and S. A. Maier, Science 337, 549 (2012), and references therein.

[32] A. I. Fernández-Domínguez, S. A. Maier, and J. B. Pendry, Phys. Rev. Lett. 105, 266807 (2010).

[33] A. I. Fernández-Domínguez, Y. Luo, A. Wiener, J. B. Pendry, and S. A. Maier, Nano Lett. 12, 5946 (2012).

[34] G. Bimonte and T. Emig, Phys. Rev. Lett. 109, 160403 (2012).

[35] R. Zhao, J. Zhou, Th. Koschny, E. N. Economou, and C. M. Soukoulis, Phys. Rev. Lett. 103, 103602 (2009).

[36] E. W. Palik, Handbook of Optical Constants of Solids I (Academic, San Diego, 1985).

[37] F. Intravaia and R. Behunin, Phys. Rev. A 86, 062517 (2012).

[38] See Supplemental Material at http://link.aps.org/ supplemental/10.1103/PhysRevLett.111.033602 for the details of the fitting.

[39] A. M. Marvin and F. Toigo, Phys. Rev. A 25, 782 (1982); 25, 803 (1982).

[40] G. L. Klimchitskaya, U. Mohideen, and V.M. Mostepanenko, Rev. Mod. Phys. 81, 1827 (2009).

[41] E. A. Power and T. Thirunamachandran, Phys. Rev. A 48, 4761 (1993). 